\title{
Proton Pump Inhibitors Therapy for Eosinophilic Esophagitis
}

\section{Javier Molina-Infante ${ }^{1 *}$, Jose Zamorano ${ }^{2}$, Maria D. Rivas ${ }^{2}$ and Miguel Fernandez-Bermejo ${ }^{1}$}

${ }^{1}$ Departments of Gastroenterology, Hospital San Pedro de Alcantara, Caceres, Spain

${ }^{2}$ Research Unit, Hospital San Pedro de Alcantara, Caceres, Spain

\begin{abstract}
Proton pump inhibitors (PPIs) are the pharmacological cornerstone for acid-related peptic disorders, especially gastroesophageal reflux disease (GERD), on account of their powerful suppression of gastric acid secretion. GERD is the most common esophageal disease, with approximately $10-20 \%$ of adult Western population affected, mainly males between 20 and 50 yrs-old. Eosinophilic esophagitis (EoE) is an emerging immuno/antigen-mediated disorder, characterized by esophageal symptoms and eosinophil-predominant inflammation. It is an allergic condition of growing recognition and prevalence, so much so that it is currently recognized as the second cause of chronic esophagitis after GERD. EoE usually presents in allergic males under $40 \mathrm{yrs}$-old, so GERD and EoE coexist frequently in adult patients. Initially, both disorders were distinguished since EoE was, by definition, unresponsive to PPIs. However, case reports and small series since 2006 have progressively shown patients with clinical, endoscopic and histological data consistent with EoE having complete remission on PPIs. Notwithstanding the fact that these patients have been historically classified as having mislabelled GERD, recent evidence has made way for a new potential phenotype of EoE (PPI-responsive esophageal eosinophilia), even in patients with normal acid exposure. Furthermore, more confusion has been added to the debate since GERD has been recently redefined as a cytokinemediated disorder rather than acid-injury mediated. As such, PPIs might also affect GERD-related or EoE-related esophageal eosinophilia through immunomodulatory mechanisms beyond acid suppression. This report intends to update the available evidence on PPI-responsive esophageal eosinophilia and briefly review the mechanisms whereby PPIs might exert potential anti-inflammatory effects on allergic diseases such as EoE.
\end{abstract}

Keywords: Eosinophilic esophagitis; Proton pump inhibitors; Gastroesophageal reflux disease; Inflammation; Anti-inflammatory agents

Abbreviations: EoE: Eosinophilic Esophagitis; GERD: Gastroesophageal Reflux Disease; PPIs: Proton Pump Inhibitors

\section{Eosinophilic Esophagitis (EoE)}

Eosinophilic esophagitis (EoE) is a recently recognized but increasing expanding chronic immune/antigen-mediated disorder, confined to the esophagus, characterized clinically by esophageal dysfunction (mainly dysphagia and food impaction) and histologically by eosinophil-predominant inflammation (usually a minimum of 15 eosinophils per high power filed (eo/HPF)) [1]. In fact, scientific publications have nearly doubled since 2007 and increased recognition has led to a steady increase in prevalence [1]. It is currently considered the second cause of chronic esophagitis after gastroesophageal reflux disease (GERD).

Unlike all other segments of the gastrointestinal tract, the esophagus is normally devoid of eosinophils, so the finding of esophageal eosinophilia denotes pathology. However, the presence of esophageal eosinophils is not specific and may occur in a variety of disorders including GERD, EoE, eosinophilic gastroenteritis, celiac disease, achalasia, inflammatory bowel disease, infection, hypereosinophilic syndrome, vasculitis, drug and/or iatrogenic-induced states such as caustic injury multiple convulsive therapy syndrome and immunosuppression especially following solid organ transplantation $[2,3]$. The diagnosis of EoE requires elimination of other causes of esophagitis, especially GERD, the most prevalent esophageal disorder affecting $10-30 \%$ of Western population. In the first consensus guidelines, it was initially established that EoE could be distinguished from GERD by either a normal esophageal $\mathrm{pH}$ monitoring or persistent esophageal eosinophilia despite adequate high-dose acid suppressive therapy [2]. However, this recommendation has not been subsequently applied or validated in clinical practice. Indeed, two recent surveys identified that only one third fulfilled strictly consensus recommendations in both pediatric and adult EoE patients [4,5]. In line with this clinical practice, growing evidence during the last five years has shown the existence of symptomatic patients who met clinical, endoscopic and histological criteria for EoE with complete clinicopathologic remission on PPI therapy [6-12]. Of note, 2011 updated EoE guidelines have highlighted as a major addition to prior knowledge the description of a new potential disease phenotype, the PPI-responsive esophageal eosinophilia [1]. So far now, EoE is considered an allergic disorder which should remit with dietary exclusion, topical steroids or both. However, evolving evidence is consistent with the existence of a significant proportion of patients with suspected EoE that achieve clinicopathological remission on PPI therapy. While such remission is the therapeutic goal in EoE, regardless of the underlying mechanisms, long-term steroid or elimination diet therapy should be discouraged in this subset of patients

\section{Proton Pump Inhibitors-Responsive Esophageal Eosinophilia: Clinical Evidence}

In 2006, Ngo et al. reported a series of three cases (two pediatric and one adult), fulfilling symptoms (refractory GERD, dysphagia, food impaction), endoscopic findings (thickened folds, ringlike contraction,

*Corresponding author: Javier Molina-Infante, MD, Department of Gastroenterology, Hospital San Pedro de Alcantara, C/ Pablo Naranjo s/n 10001 Caceres, Caceres, Spain, Tel: 0034 627430248; Fax: 0034927621545; E-mail: xavi_molina@hotmail.com

Received November 28, 2011; Accepted December 19, 2011; Published December 24, 2011

Citation: Molina-Infante J, Zamorano J, Rivas MD, Fernandez-Bermejo M (2011) Proton Pump Inhibitors Therapy for Eosinophilic Esophagitis. J Aller Ther S8:002. doi:10.4172/2155-6121.S8-002

Copyright: (C) 2011 Molina-Infante J, et al. This is an open-access article distributed under the terms of the Creative Commons Attribution License, which permits unrestricted use, distribution, and reproduction in any medium, provided the original author and source are credited. 
furrows, exudates) and histological (>20 eo/HPF) data consistent with EoE which fully reverted to normal after PPI therapy [6]. The authors concluded that these patients were likely to have mislabelled GERD and that large number of eosinophils can be found related to peptic esophagitis. In agreement with this study, Rodrigo et al reported in 2008 a retrospective series of 40 out of 3648 patients with esophageal eosinophilia (>20 eo/HPF) [7]. 28 patients (70\%) had a final diagnosis of GERD after careful evaluation of clinical, endoscopic, manometric and $\mathrm{pH}$ monitoring information. The main conclusion drawn was that esophageal eosinophilia was not specific of EoE. In 2009, Dranove et al. published an interesting retrospective series of 43 pediatric patients with esophageal eosinophilia ( $>15 \mathrm{eo} / \mathrm{HPF}$ ) who received PPI therapy [8]. In this report, 17 patients $(40 \%)$ achieved clinical and histological $(<5 \mathrm{eo} /$ HPF) remission on PPIs. Interestingly, PPI-response was identified in $45 \%$ and $41 \%$ of patients with normal and abnormal $\mathrm{pH}$ esophageal monitoring, respectively, thereby questioning the role of esophageal acid exposure to predict response to PPI therapy. These findings raised the possibility of a PPI-responder phenotype different from EoE among patients with esophageal eosinophilia. In 2009, Sayej et al. reported a retrospective series of 69 pediatric patients with $\geq 15 \mathrm{eo} / \mathrm{HPF}$, of whom 36 (52\%) were treated with high-dose PPI therapy [9]. 14 out of those 36 patients $(39 \%)$ had clinical and histological remission $(<5$ eo/HPF) on PPIs, emphasizing the existence of a significant proportion of PPI-responders among patients with suspected EoE. It was in 2010 that it was published the first randomized controlled trial comparing esomeprazole ( $40 \mathrm{mg}$ a day) and topical fluticasone ( $440 \mathrm{mcg}$ bid) in 30 adult patients with $\geq 15 \mathrm{eo} / \mathrm{HPF}[10]$. There were no differences regarding improvement in average dysphagia score or eosinophilic infiltration between both groups. Partial histological response $(<15$ eo/HPF) and complete remission $(<5$ eo/HPF) were accomplished in $31 \%$ and $15 \%$ in the fluticasone group and in $50 \%$ and $33 \%$ in the PPI group. These results highlighted that both drugs were equally effective in decreasing esophageal eosinophilic infiltration and this was attributable to a high proportion of patients with coexistent GERD (56\%), documented by pH monitoring, in the cohort. In 2011, our group reported the first prospective series addressing the role of systematic high-dose PPI therapy in adult patients with $\geq 15$ eo/HPF [11]. Upper-mid esophageal biopsies were taken in 712 consecutive patients with upper GI symptoms, of whom 35 patients (5\%) had esophageal eosinophilia. All of these patients with $\geq 15 \mathrm{eo} / \mathrm{HPF}$ underwent clinical, endoscopic and histological follow-up after high-dose PPI therapy (rabeprazole $20 \mathrm{mg}$ b.i.d) for 8 weeks. Overall, $75 \%$ of patients (26/35) achieved clinical and histological $(<5$ eo/HPF) remission on PPI therapy. Excluding patients with a GERD profile (heartburn as main symptom, erosive esophagitis in endoscopy), we observed that even $50 \%$ of EoE profile patients were PPI-responders. Of note, remission on PPI therapy occurred among patients with normal and abnormal $\mathrm{pH}$ monitoring, in agreement with that reported by Dranove et al. [9]. As such, pathologic acid exposure is not predictive of PPI response, raising the possibility of a new potential PPI-responsive EoE phenotype without GERD. Abe et al. [12] reported lately the first retrospective case series from Japan involving 12 patients. 6 out of 12 were treated with PPIs, of whom 3 patients (50\%) achieved clinical and histological remission. Finally, a more recent case series has reported four pediatric EoE patients with transient PPI responsive esophageal inflammation, including eosinophils and mast cells [13].

\section{Proton pump inhibitors}

Proton pump inhibitors (PPIs) are substituted benzimidazoles targeted to inhibit the proton pump of the parietal cell $(\mathrm{H}-\mathrm{K}-$ ATPase), which comprises the final pathway by which hydrochloric acid is secreted into the gastric lumen. All PPIs molecules are weak protonatable pyridines that accumulate specifically and selectively in the secretory canaliculus, the highly acid space of the gastric parietal cell. PPIs are prodrugs which become activated within that acid environment to a reactive species, the thiophilic sulfenamide. Then, this reactive species binds covalently with sulfhydryl groups of cysteines in the $\mathrm{H}^{+} / \mathrm{K}^{+}$ATPase (cysteine alkylation) irreversibly inactivating the pump molecules $[14,15]$, resulting in a specific and long-lasting impairment of acid suppression, regardless of other stimulating factors. PPIs are the most effective acid inhibitors currently available and are the most widely prescribed class of gastrointestinal medications [15]. Currently, PPIs are the treatment of choice for all acid-related disorders including peptic ulcer disease, gastroesophageal reflux disease (GERD), eradication of Helicobacter pylori, prevention of ulcers associated with non-steroidal anti-inflammatory drugs (NSAIDs) and ZollingerEllison syndrome.

\section{Antiinflammatory effects of PPIs: Evidence from animal models}

The PPIs are weak bases that become active in an acidic environment by means of blocking the p-type $\mathrm{H}^{+}, \mathrm{K}^{+}$ATPases of parietal cells, final responsible for acid secretion into the gastric lumen. Non-gastric cells, like neutrophils and endothelial cells, also have $\mathrm{H}^{+}, \mathrm{K}^{+}$ATPases that pump acid into the extracellular space and into intracellular organelles like lysosomes. An acidic microenvironment, in fact, is critical for survival in inflammation animal models [16]. When neutrophils are activated by chemotactic factors, those vacuolar $\mathrm{H}^{+}$ATPases pump $\mathrm{H}^{+}$into the phagolysosome. This lysosomal acidification is key for neutrophil's oxidative burst, the secretion of reactive oxygen species (ROS) and activation of adhesion molecules [17-20]. Growing evidence has lately highlighted a marked impairment on neutrophil function by PPIs in experimental models. In the 1990s, omeprazole treatment of the neutrophils was found to result both in elevated intralysosomal $\mathrm{pH}$ and in inhibition of the oxidative burst [21], whereas omeprazole [22] and lansoprazole [23] were shown to inhibit neutrophil superoxide generation and oxidative burst of human neutrophils, respectively, after neutrophil activation in the stomach with bacterial substances. Lansoprazole [22] also decreased the gastric mucosal levels of myeloperoxidase (an index of neutrophil infiltration) in patients with $H$. pylori gastritis. Agastya et al. [23] found that acid-activated omeprazole inhibited the acidification of phagolysosomes in human neutrophils, and that this phenomenon was associated with impaired ability of the neutrophil to phagocytose yeast. In healthy volunteers, orally administered omeprazole caused a significant decrease in neutrophil production of ROS [20-24]. Furthermore, omeprazole and lansoprazole have been shown to inhibit in vitro the expression of CD11b and CD18 by human neutrophils as well as neutrophil-dependant adhesion to endothelial cells [25]. Similarly, PPIs inhibited the expression of intercellular adhesion molecule-1 (ICAM-1), vascular cell adhesion molecule-1 (VCAM-1) and endothelial-dependent neutrophil adhesion in human umbilical vein endothelial cells stimulated with interleukin$1 \mathrm{~b}$ [26]. In addition, lansoprazole also has been found to inhibit the expression of ICAM-1 by human tracheal epithelial cells in culture [27] and to decrease the number of peripheral blood mononuclear cells that express ICAM-1 in human volunteers [28]. Overall, this impairment in neutrophil function has been related to the presence of vacuolar (v-type) $\mathrm{H}^{+}$ATPases in neutrophils, which might be potentially susceptible to inhibition by PPIs. Nevertheless, the precise mechanisms underlying PPI effects on neutrophil function remain to be elucidated.

As for pro-inflammatory cytokines, PPIs may exert antiinflammatory effects by inhibiting the production of these cytokines 
that recruit inflammatory cells to diseased tissues. Omeprazole and lansoprazole were shown to significantly block gastric mucosal production of IL-8, a potent neutrophil chemoattractant, after stimulation with H. Pylori [29]. In rats treated with indomethacin, lansoprazole significantly decreased the production of cytokineinduced neutrophil chemoattractant-1 (CINC-1, a rat homologue of IL-8) by the small intestine [30], whereas it decreased levels of proinflammatory cytokines including IL-6, IL- 8 and TNF- $\alpha$ in cultured human tracheal epithelial cells [31]. The mechanisms underlying the PPI-induced decrease in pro-inflammatory cytokine production by epithelial and endothelial cells are not clear, albeit we should be aware of the fact that response to PPIs should not always be construed as proof for an underlying acid-peptic disorder.

\section{Proton pump inhibitors-responsive esophageal eosinophilia: Evidence from animal models}

Up to now, few data are available in this field. In 2009, our group reported a novel anti-inflamamatory effect of PPIs (including omeprazole, lansoprazole and esomeprazole) in murine asthma [30]. To begin with, it was demonstrated that PPIs inhibit in vitro IL-4 and IL-13 signaling STAT6, which is a well known pathogenic pathway in Th2 allergic diseases, like asthma and EoE. Furthermore, PPI therapy in asthmatic mice reduced significantly the presence of inflammatory cells in bronchoalveolar lavage fluid and lung sections, including eosinophils. Overall, these findings raised the possibility that the benefitial effects of PPIs in allergic disease may be, at least in part, mediated through the inhibition of these pathways. Our results were corroborated in a further interesting investigation which is only published in abstract form so far today [31]. Two different esophageal squamous epithelial cell lines from patients with EoE were treated with either IL-13 and IL-14 in the presence and absence of omeprazole. Both interleukins increased the secretion of eotaxin-3, which could be blocked by omeprazole treatment. These findings suggest that PPIs can have anti-inflammatory actions in EoE independent of their effects on acid-secretion, and cast doubt on the assumption that a positive response to PPI therapy distinguishes EoE from GERD.

\section{The controversial relationship between GERD and EoE}

Since both GERD and EoE can be associated with mild esophageal eosinophilia and may share common symptoms like heartburn and dysphagia, it can be difficult to distinguish between the two disorders. Moreover, both diseases affect predominantly males on their $2^{\text {nd }}-4^{\text {th }}$ decade and indeed coexist (38-56\%) as evidenced by cohorts with persistent symptoms and esophageal eosinophilia on PPI therapy in spite of abnormal pH testing [12,32-33]. In the first consensus EoE guidelines, it was initially established that EoE could be distinguished from GERD by persistent esophageal eosinophilia despite adequate high-dose acid suppressive therapy [2]. However, Spechler et al. [34] in an elegant clinical reviews series, raised the possibility of a complex interaction between EoE and GERD and discussed potential mechanisms whereby GERD may be a mimic of EoE, coexist with it, contribute to it, or conversely, EoE may contribute to GERD. In this regard, a number of clinical, endoscopic and histological features have been proposed to distinguish EoE from GERD. Patients with EoE are more likely to be male, younger than 45 years, to have dysphagia and endoscopic features of EoE (ring, furrows and exudates) and less likely to have hiatal hernia or heartburn [35]. Similarly, a recent report has proven a high sensitivity (91\%) and specificity (100\%) for diagnosing EoE before endoscopy using a simplified equation: peripheral eosinophilia + history of food impaction + PPI refractory heartburn
[36]. As for histological features, eosinophilic microabscesses [37], tryptase mast cell staining [38] and levels of eotaxin-3 in esophageal biopsies [39] have been suggested to favour EoE, albeit further studies are needed to set a delimited distinction between EoE and GERD.

Evolving research [6-13] and updated 2011 guidelines [1], as mentioned above, have highlighted that a clear distinction between GERD and EoE may be difficult, even more upon the existence of a novel EoE phenotype, the PPI-responsive esophageal eosinophilia. These patients are undistinguishable from EoE patients in clinical, endoscopic and histological terms. Whether they represent mislabelled allergic GERD or a real EoE phenotype merits further investigation upon genetic background. Overall, the most important lesson we have learned is that a favourable response to PPI therapy does not preclude a diagnosis of EoE. In addition, the same group of investigators from Dallas published recently a striking paper in which they demonstrate that gastroesophageal induced reflux in an animal model causes esophagitis through a cytokine-mediated mechanism rather than caustic acid-injury [40]. For decades, the prevailing concept of GERD has been that esophageal injury is caused by the toxic direct effects of refluxed gastric acid, causing the death of surface epithelial cells, which would evoke an acute inflammatory response with epithelial infiltration by neutrophils. In this elegant paper, the authors observe indeed the opposite sequence of events. At day 3 after surgical induction of reflux, there was no surface damage and the first inflammatory sign detected was a lymphocitic infiltration of the submucosa, that progressed to the mucosal surface, closely related to an increase in the secretion of IL-8 and IL-1 $\beta$. Interestingly, mucosal erosions did not appear until postoperative week 4 , which is consistent with reflux esophagitis develops primarily as an immune-related injury rather than as a caustic chemical injury. Since EoE has been recently redefined as a chronic immune-mediated disease as well, one can speculate that PPI-responsive esophageal eosinophilia may be represented by GERD provoking an aberrant inflammatory response in patients with genetic or clinical allergic background, mimicking EoE, but responsive to PPIs. Undoubtedly, this work opens up an attractive field of investigation for the ongoing years.

\section{References}

1. Liacouras CA, Furuta GT, Hirano I, Atkins D, Attwood SE, et al. (2011) Eosinophilic esophagitis: updated consensus recommendations for children and adults. J Allerg Clin Immunol 128: 3-20.

2. Furuta GT, Liacouras CA, Collins MH, Gupta SK, Justinich C, et al. (2007) Eosinophilic esophagitis in children and adults: a systematic review and consensus recommendations for diagnosis and treatment. Gastroenterology 133: $1342-1363$

3. Rothemberg ME (2009) Biology and treatment of eosinophilic esophagitis Gastroenterology137: 1238-1249.

4. Peery AF, Shaheen NJ, Dellon ES (2010) Practice patterns for the evaluation and treatment of eosinophilic esophagitis. Aliment Pharmacol Ther 32: 1373 1382.

5. Spergel JM, Book WM, Mays E, Song L, Shah SS, et al. (2011) Variation in prevalence, diagnostic criteria, and initial management options for eosinophilic gastrointestinal diseases in the United States. J Pediatr Gastroenterol Nutr 52 300-306.

6. Ngo P, Furuta GT, Antonioli DA, Fox VL (2006) Eosinophils in the esophagus: peptic or allergic eosinophilic esophagitis? Case series of three patients with esophageal eosinophilia. Am J Gastroenterol 101: 1666-1670.

7. Rodrigo S, Abboud G, Oh D, DeMeester SR, Hagen J, et al. (2008) High intraepithelial eosinophil counts in esophageal squamous epithelium are not specific for eosinophilic esophagitis in adults. Am J Gastroenterol 103: 435-442.

8. Dranove JE, Horn DS, Davis MA, Kernek KM, Gupta SK (2009) Predictors 
of response to proton pump inhibitor therapy among children with significant esophageal eosinophilia. J Pediatr 154: 96-100.

9. Sayej WN, Patel RA, Baker RD, Tron E, Baker SS (2009) Treatment with highdose proton pump inhibitors helps distinguish eosinophilic esophagitis from noneosinophilic esophagitis. J Pediatr Gastroenterol Nutr 49: 1-7.

10. Peterson KA, Thomas KL, Hilden K, Emerson LL, Wills JC, et al. (2010) Comparison of esomeprazole to aerosolized, swallowed fluticasone for eosinophilic esophagitis. Dig Dis Sci 55: 1313-1319.

11. Molina-Infante J, Ferrando-Lamana L, Ripoll C, Hernandez-Alonso M, Mateos $\mathrm{JM}$, et al. (2011) Esophageal eosinophilic infiltration responds to proton pump inhibition in most adults. Clin Gastroenterol Hepatol 9: 110-117.

12. Abe Y, lijima K, Ohara S, Koike T, Ara N, et al. (2011) A Japanese case series of 12 patients with esophageal eosinophilia. J Gastroenterol 46: 25-30.

13. Dohil R, Newbury RO, Aceves S (2011) Transient PPI responsive esophagea eosinophilia may be a clinical sub-phenotype of pediatric eosinophilic esophagitis. Dig Dis Sci: in press.

14. Kedika RR, Souza RF, Spechler SJ (2009) Potential anti-inflammatory effects of proton pump inhibitors: a review and discussion of the clinical implications. Dig Dis Sci 54: 2312-2317.

15. Schubert ML, Peura DA (2008) Control of gastric acid secretion in health and disease. Gastroenterology 134: 1842-1860.

16. Namazi MR, Jowkar F (2008) A succint review of the general and pharmacological effects of proton pump inhibitors. J Clin Pharm Ther 33: 215 217

17. Ritter M, Schratzberger P, Rossmann H, Wöll E, Seiler K, et al. (1998) Effect of inhibitors of $\mathrm{Na}+/ \mathrm{H}+-$-exchange and gastric $\mathrm{H}+/ \mathrm{K}+$ ATPase on cell volume, intracellular $\mathrm{pH}$ and migration of human polymorphonu-clear leucocytes. $\mathrm{Br} \mathrm{J}$ Pharmacol 124: 627-638.

18. Lafourcade C, Sobo K, Kieffer-Jaquinod S, Garin J, van der Goot FG (2008) Regulation of the V-ATPase along the endocytic pathway occurs through reversible subunit association and membrane localization. PLoS ONE 3: e2758.

19. Luciani F, Spada M, De Milito A, Molinari A, Rivoltini L, et al. (2004) Effect of proton pump inhibitor pretreatment on resistance of solid tumors to cytotoxic drugs. J Natl Cancer Inst 96: 1702-1713.

20. Wandall JH (1992) Effects of omeprazole on neutrophil chemotaxis, supe oxide production, degranulation and translocation of cytochrome b-245. Gut 33: $617-621$

21. Suzuki M, Mori M, Miura S, Suematsu M, Fukumura D, et al. Omeprazole attenuates oxygen-derived free radical production from human neutrophils. Free Radic Biol Med 21: 727-731.

22. Suzuki M, Nakamura M, Mori M, Miura S, Tsuchiya M, et al. (1995) Lansoprazole inhibits oxygen derived free radical production from neutrophils activated by Helicobacter pylori. J Clin Gastroenterol 20: 93-96.

23. Agastya G, West BC, Callahan JM (2000) Omeprazole inhibits phagocytosis and acidification of phagolysosomes of normal human neutrophils in vitro. Immunopharmacol Immunotoxicol 22: 357-372.

24. Zedtwitz-Liebenstein K, Wenisch C, Patruta S, Parschalk B, Daxböck F, et al (2002) Omeprazole treatment diminishes intra- and extracellular neutrophi reactive oxygen production and bactericidal activity. Crit Care Med 30: 11181122.

25. Yoshida N, Yoshikawa T, Tanaka Y, Fujita N, Kassai K, et al. (2000) A new mechanism for anti-inflammatory actions of proton pump inhibitors-inhibitory effects on neutrophil-endothelial cell interactions. Aliment Pharmacol Ther 14 74-81

26. Sasaki T, Yamaya M, Yasuda H, Inoue D, Yamada M, et al. (2005) The proton pump inhibitor lansoprazole inhibits rhinovirus infection in cultured human tracheal epithelial cells. Eur J Pharmacol 509: 201-210.

27. Ohara T, Arakawa T (1999) Lansoprazole decreases peripheral blood monocytes and intercellular adhesion molecule-1-positive mononuclear cells. Dig Dis Sci 44: 1710-1715.

This article was originally published in a special issue, Eosinophilic disorders and Allergic disorders handled by Editor(s). Dr. Yoshiyuki Yamada, Gunma Children's Medical Center, Japan
28. Handa O, Yoshida N, Fujita N, Tanaka Y, Ueda M, et al. (2006) Molecular mechanism envolved in anti-inflammatory effects of proton pump inhibitors. Inflamm Res 55: 476-480.

29. Kuroda M, Yoshida N, Ichikawa H, Takagi T, Okuda T, et al. (2006) Lansoprazole, a proton pump inhibitor, reduces the severity of indomethacin-induced rat enteritis. Int J Mol Med 17: 89-93.

30. Cortes JR, Rivas MD, Molina-Infante J, Gonzalez-Nuñez MA, Perez-G M, et al. (2009) Omeprazole inhibits IL-4 and IL-13 signaling signal transducer and activator of transcription 6 activation and reduces lung inflammation in murine asthma. J Allergy Clin Immunol 124: 607-610.

31. Zhang X, Cheng E, Huo X, Yu C, Hormi-Carver KK, et al. (2010) In esophageal squamous epithelial cell lines from patients with eosinophilic esophagitis (EoE) omeprazole blocks the stimulated secretion of eotaxin-3: a potential antiinflammatory effect of omeprazole in EoE that is independent of acid inhibition. Gastroenterology 138: S-122.

32. Remedios M, Campbell C, Jones DM, Kerlin P (2006) Eosinophilic esophagitis in adults: clinical, endoscopic, histologic findings, and response to treatment with fluticasone propionate. Gastrointest Endosc 63: 3-12.

33. Shah A, Kagalwalla AF, Gonsalves N, Melin-Aldana H, Li BU, et al. (2009) Histopathologic variability in children with eosinophilic esophagitis. Am J Gastroenterol 104: 716-721.

34. Spechler SJ, Genta RM, Souza RF (2007) Thoughts on the complex relationship between gastroesophageal reflux disease and eosinophilic esophagitis. Am Gastroenterol 102: 1301-1306.

35. Dellon ES, Gibbs WB, Fritchie KJ, Rubinas TC, Wilson LA, et al. (2009) Clinical, endoscopic and histologic findings distinguish eosinophilic esophagitis from reflux disease. Clin Gastroenterol Hepatol 7: 1305-1313.

36. von Arnim U, Wex T, Rohl FW, Neumann H, Küster D, et al. (2011) Identification of clinical and laboratory markers for predicting eosinophilic esophagitis in adults. Digestion 84: 323-327.

37. Parfitt JR, Gregor JC, Suskin NG, Jawa HA, Driman DK (2006) Eosinophilic esophagitis in adults: disitinguishing features from gastroesophageal reflux disease. Mod Pathol 19: 90-96.

38. Dellon ES, Chen X, Miller CR, Fritchie KJ, Rubinas TC, et al. (2011) Tryptase staining of mast cells may differentiate eosinophilic esophagitis from gastroesophageal reflux disease. Am J Gastroenterol 106: 264-271.

39. Bhattacharya B, Caristen J, Sabo E, Kethu S, Meitner P, et al. (2007) Increased expression of eotaxin-3 distinguishes between eosinophilic esophagitis and gastroesophageal reflux disease. Hum Pathol 38: 1744-53.

40. Shouza RF, Huo X, Vittal M, Schuler CM, Carmack SW, et al. (2009) Gastroesophageal reflux might cause esophagitis through a cytokine-mediated mechanism rather than caustic acid injury. Gastroenterology 137: 1776-1784. 